\title{
White Matter Microstructure and Apathy Level in Amnestic Mild Cognitive Impairment
}

\author{
Claudia Cacciari ${ }^{\mathrm{a}}$, Marta Moraschi ${ }^{\mathrm{a}, \mathrm{b}}$, Margherita Di Paola $^{\mathrm{a}, \mathrm{c}}$, Andrea Cherubini $^{\mathrm{a}}$, Maria Donata Orfei ${ }^{\mathrm{a}}$, \\ Federico Giove $^{\mathrm{a}, \mathrm{b}}$, Bruno Maraviglia ${ }^{\mathrm{a}, \mathrm{d}}$, Carlo Caltagirone ${ }^{\mathrm{a}, \mathrm{e}}$ and Gianfranco Spalletta ${ }^{\mathrm{a}, *}$ \\ a IRCCS Santa Lucia Foundation, Rome, Italy \\ bMARBILab, "Enrico Fermi" Center, Rome, Italy \\ ${ }^{\mathrm{c}}$ Department of Internal Medicine and Public Health, University of L'Aquila, L'Aquila, Italy \\ ${ }^{\mathrm{d}}$ Department of Physics, "Sapienza" University of Rome, Rome, Italy \\ e Department of Neuroscience, University "Tor Vergata”, Rome, Italy
}

\begin{abstract}
In this study, we assessed white matter microstructural deficit correlates of apathy level in 20 patients with amnestic mild cognitive impairment by means of diffusion tensor imaging. Mean diffusivity correlated positively with apathy level in the right temporal portion of the uncinate, middle longitudinal and inferior longitudinal fasciculi and in the parathalamic white matter, the fornix and the posterior cingulum of the right hemisphere. Fractional anisotropy results confirmed evidence of disconnection associated with apathy in all white matter areas except the middle longitudinal fasciculus. These results support the view that alterations in the neural mechanisms underlying apathy level occur in the early phase of degenerative dementias.
\end{abstract}

Keywords: Apathy, diffusion tensor imaging, microstructure, mild cognitive impairment, MRI, white matter

\section{INTRODUCTION}

Mild cognitive impairment (MCI) refers to the transitional state between normal aging and dementia. Apathy is the most frequent and severe behavioral symptom in both MCI [1] and Alzheimer's disease (AD) [2, 3 ] and may characterize a subset of MCI subjects at higher risk of progression to dementia [4-7]. The functional neuroanatomy of apathy has been studied primarily in AD patients, where it was found that bilateral frontal, temporal, and cingulate area hypoperfusion and hypometabolism are associated with apathy severity [8-12]. Several structural neuroimaging studies also demonstrated an association between apathy and loss of integrity of frontal regions in AD [13,14]. Taken together, these results demonstrate that different brain

${ }^{*}$ Correspondence to: Gianfranco Spalletta, MD, PhD, I.R.C.C.S. Santa Lucia Foundation, Laboratory of Clinical and Behavioral Neurology, Via Ardeatina, 306-00179 Rome, Italy. Tel./Fax: +3906 51501575; E-mail: g.spalletta@hsantalucia.it. areas are implicated in the pathophysiology of apathy in $\mathrm{AD}$ and suggest that circuits, rather than individual structures, may be disrupted in apathetic patients.

However, as far as we know, no studies have investigated the white matter microstructural correlates of apathy in MCI patients. Thus, we aimed to analyze the association between local white matter tissue microstructure, assessed by diffusion tensor imaging (DTI), and apathy level in a sample of amnestic (a-) MCI patients.

\section{MATERIALS AND METHODS}

Twenty patients with a diagnosis of a-MCI were recruited for this study. Inclusion was based on a diagnosis of a-MCI according to the Petersen et al. [15] criteria: complaint of defective memory, normal activities of daily living, normal global cognitive function, abnormal memory function for age, and absence of dementia. Exclusion criteria were the following: major medical illness (e.g., hematological or oncological; vitamin B12 or folate deficiency; pernicious anemia; clin- 
ically significant and unstable active gastrointestinal, renal, hepatic, endocrine or cardiovascular system disease); newly treated hypothyroidism; comorbidity of primary psychiatric or neurological disorders; known or suspected history of alcoholism or drug dependence and abuse during lifetime; focal parenchymal abnormalities; excessive atrophy which could impede correct MRI registration and normalization. In addition, white matter lesions were considered present if they were hyperintense on proton-density (PD)/T2-weighted images. We included only subjects who, in the opinion of an expert neuroradiologist, had no lesions. Thus, even one small lesion was considered as an exclusion criterion.

All subjects gave their written informed consent to participate in the study, which was approved by the Local Ethics Committee.

All examinations were performed on a 3 Tesla MR scanner (Siemens Magnetom Allegra) using a standard quadrature bird-cage head coil. DTI data acquisition was performed applying diffusion sensitizing gradients, with $b=1000 \mathrm{~s} / \mathrm{mm}^{2}$, along 12 non-coplanar directions; one additional scan without diffusion weighting ( $b=0 \mathrm{~s} / \mathrm{mm}^{2}$ ) was also obtained. Three DTI series (inplane resolution $=1.5 \times 1.5 \mathrm{~mm}^{2}$, slice thickness $=3$ $\mathrm{mm}$ ) were acquired for each subject. Anatomical $T 1-$ weighted data (in-plane resolution $=1 \times 1 \mathrm{~mm}^{2}$, slice thickness $=1 \mathrm{~mm}$ ) for tissue segmentation and conventional MRI generating proton-density (PD) and T2weighted images (in-plane resolution $=1.5 \times 1.5 \mathrm{~mm}^{2}$, slice thickness $=3 \mathrm{~mm}$ ) were also acquired to control for the presence of any radiological alterations.

Before performing statistical analyses, the DTI datasets underwent multiple pre-processing steps, performed with selected routines from Analysis of Functional NeuroImages (AFNI) [16] and Statistical Parametric Mapping (SPM5) packages [17]. We performed a two-stage co-registration on each of the three DTI datasets to partially correct for both subject movements (affine transformation to the $b_{0}$ image) and eddycurrents related distortions (non-linear b-spline to the $\mathrm{b}_{0}$ image) [18]. A moderate anisotropic smoothing (3 iterations) was applied [16]; parameters were specifically optimized to keep blurring extremely low [19]. The diffusion tensor was evaluated voxel-by-voxel with an iterative algorithm [16], which assures positive definite matrices; mean diffusivity (MD) and fractional anisotropy (FA) maps were derived from the tensor. The FA and MD maps were averaged across the three sessions and subsequently normalized using an optimized protocol [20], which resulted in a tailored tem- plate in Montreal Neurological Institute (MNI) space. Then, the MNI coordinates were transformed into Talairach Tournox coordinates [21]. To reduce any biases induced by head size variability, the brain volume (BV) value was estimated [17] and used as a covariate variable in subsequent statistical analyses.

To diagnose MCI, the Mental Deterioration Battery (MDB) [22] was administered to obtain information about the functioning of different cognitive domains. The MDB is a standardized and validated neuropsychological battery comprising seven neuropsychological tests from which eight performance scores can be derived. Four test scores assess verbal stimuli and four visuospatial abilities. Tests were also given to assess long-term visual memory [23,24], complex constructional praxis [23,24], semantic fluency [25], attention control and inhibition function [26], set-shifting and cognitive flexibility [27]. Impairment in individual cognitive domains was defined using normative data [22].

Apathy level was assessed using an Italian version (translated into Italian and back translated into English for semantic congruency in both American and Italian sites) of the Dementia Apathy Interview and Rating (DAIR), a 16-item structured interview [28]. Furthermore, the Dysphoria subscale of the CERAD Behavior Rating Scale for Dementia [29] was administered to assess severity of depression.

To test the hypothesis that specific white matter microstructural changes in the brain are critically related to apathy level in a-MCI patients, we used multiple regression analysis, performed voxelwise with SPM5. The correlation model for MD maps (considered as dependent variable) included DAIR scores, age, and BV as independent variables. Statistical analyses were circumscribed to a mask obtained by thresholding the average FA map to 0.2 so as to include only white matter areas [30]. Statistical significance of the correlation between diffusion parameters and DAIR scores was tested at a voxel level by treating age and BV as confounding covariates. Voxels were deemed statistically significant at $p<0.05$, False Discovery Rate (FDR) corrected for multiple comparisons [31] with a minimum cluster size of 20 voxels. An additional analysis $(p<0.05$ FDR corrected [31], masked at the FA value of 0.2 ) with a minimum cluster size of 5 voxels was performed to detect even smaller structures.

Furthermore, since depression could be a confounding factor influencing apathy level, the same multiple regression analysis was performed for MD maps by adding the CERAD Dysphoria scores as confounding covariate. 


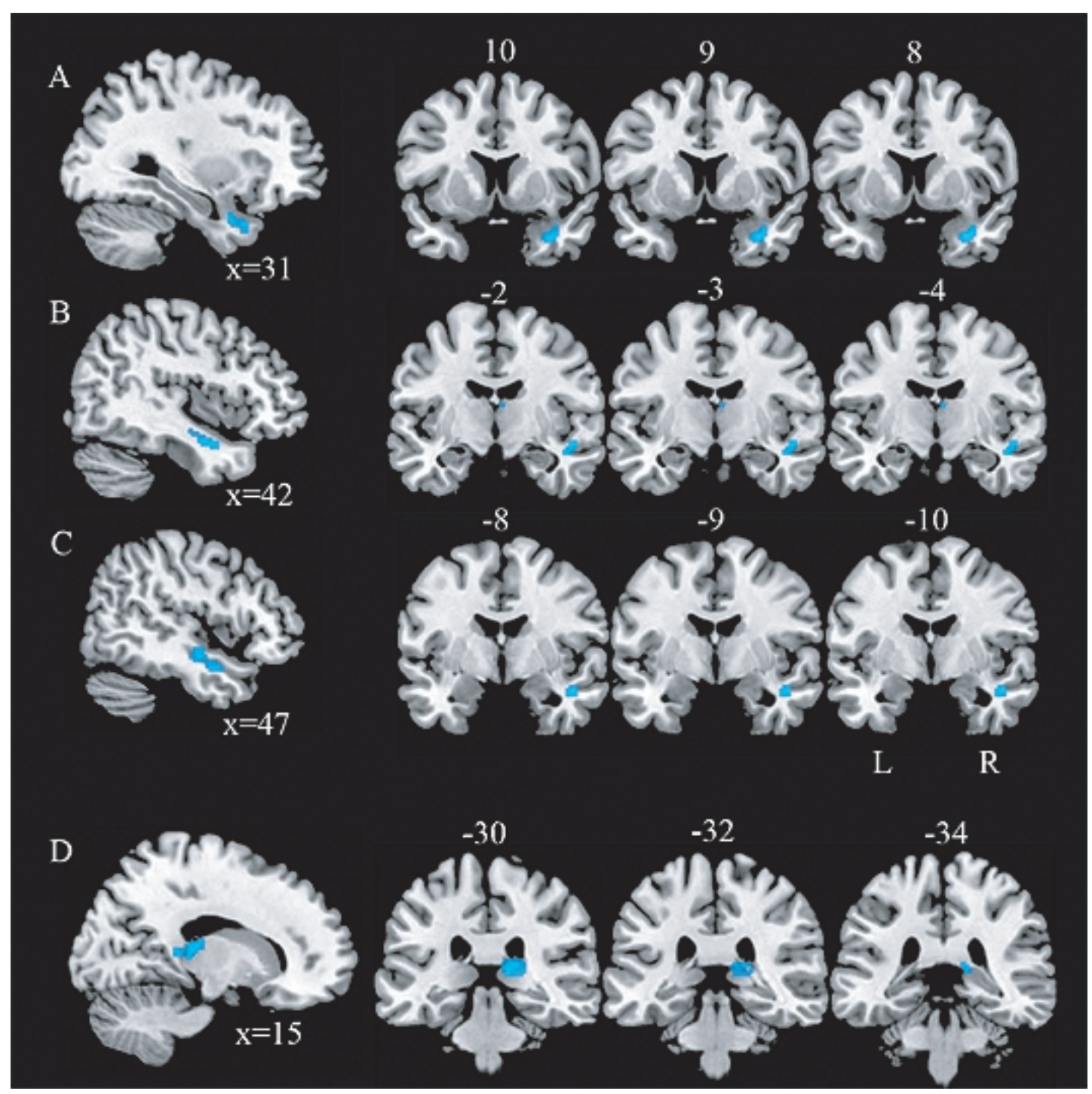

Fig. 1. Mean diffusivity (MD) correlates of apathy level in 20 amnestic MCI patients. Light blue indicates where white matter (WM) MD values are positively correlated with apathy level, as measured by the Dementia Apathy Interview and Rating. Statistically significant results are shown at $\mathrm{p}$-value $<0.05$ (False Discovery Rate corrected for multiple comparisons). $\mathrm{R}=$ right; $\mathrm{L}=$ left. A sagittal view of WM fibers is presented on the left. On the right, the coronal slices provide a better view of WM fiber position, proceeding in an anterior-posterior direction. Panel A shows the uncinate fasciculus (UF) at Talairach Tournox coordinates $(x=31$; and from $y=10$ to $y=8)$. Panel B shows the inferior longitudinal fasciculus (ILF) at Talairach Tournox coordinates $(x=42$; from $y=-2$ to $y=-4)$. Panel C shows the middle longitudinal fasciculus (MLF) at Talairach Tournox coordinates $(x=47$; from $y=-8$ to $y=-10)$. Panel D shows the medial white matter area including parathalamic white matter $(y=-30)$, fornix $(y=-32)$, and posterior cingulum $(y=-34)$. Talairach Tournox coordinates $(x=15$; from $y=-30$ to $y=-34)$.

\section{RESULTS}

We found a statistically significant positive correlation between DAIR scores and MD values in four different white matter areas using a minimum cluster size of 20 voxels.

Three of these areas were identified in the right temporal lobe. Based on the Talairach and Tournoux [21] and Schmahmann and Pandya atlases [32], these three areas were identified as the uncinate fasciculus (UF) (cluster size of 55 voxels), the middle longitudinal fasciculus (MLF) (cluster size of 36 voxels), and the inferior longitudinal fasciculus (ILF) (cluster size of 33 voxels). One additional area was found in the mesial brain region of the right hemisphere (cluster size of 31 voxels). This latter area comprised the parathalamic 


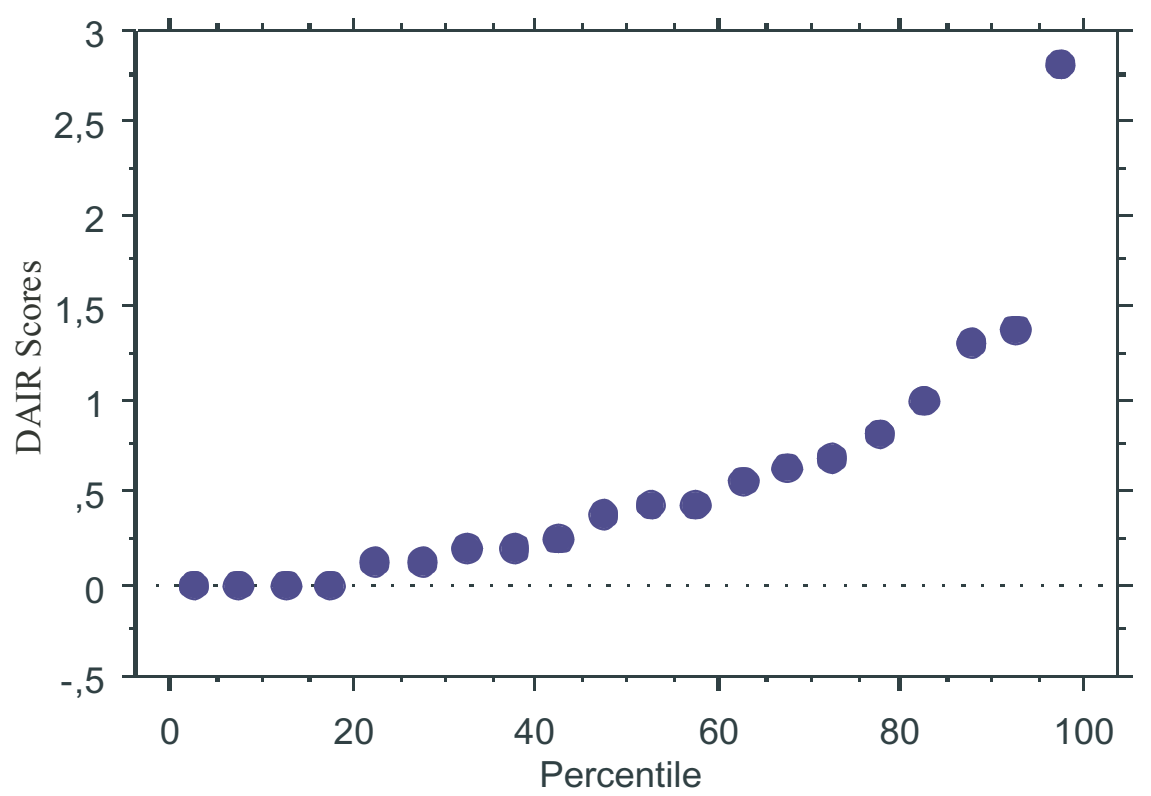

Fig. 2. Percentile plot of DAIR scores of 20 amnestic MCI patients. Dots indicate individual a-MCI patients.

white matter, the fornix and the posterior cingulum.

Considering the possibility that statistically significant correlations may be highly dependent upon scores of a single patient, we indicated the percentile plot of DAIR values of all 20 a-MCI patients. In reality, one of the patients scored much higher than the others. Thus, we explored this possibility in new correlations (one for each anatomical areas which predicted apathy level in the original sample of 20 patients) excluding the individual patient with the higher apathy severity. Results confirm that even excluding this apathy score in all new analyses, the relationships between MD values and apathy level remain highly significant $(p<0.001, r>$ 0.5 for all correlations), thus excluding the possibility of a bias induced by the outlier.

With a minimum cluster size of 5 voxels, we identified several additional white matter bilateral regions: the left superior longitudinal fasciculus (SLF) I branch (coordinates: $x=-38, y=8, z=47$; cluster size of 7 voxels); the left SLF III branch (coordinates: $x=-34$, $y=4, z=27$; cluster size of 7 voxels); the right SLF III branch (coordinates: $x=38, y=2, z=24$; cluster size of 11 voxels); the left cingulum (coordinates: $x=-7, y=4, z=36$; cluster size of 8 voxels), and the left ILF (coordinates: $x=-56, y=-26, z=13$; cluster size of 8 voxels).

In the multiple regression analysis with the CERAD Dysphoria score added to age and BV as confounding factors, we found a statistically significant positive cor- relation between DAIR scores and MD values in all previously reported white matter areas except the right MLF and the posterior cingulum. This finding indicates that depression modulates the relationship between MD and apathy level only in the latter two areas.

To support the hypothesis that white matter is also disconnected in the same areas in which it is microstructurally disrupted in association with apathy level, multiple regression analyses were performed for FA maps (using DAIR scores as a regressor) in those regions in which a positive correlation between MD and DAIR scores was found. Age and BV were treated as confounding covariates. We found a statistically significant ( $p<0.05$ uncorrected, minimum cluster size of 5 voxels) negative correlation between DAIR scores and FA values in all white matter areas except the MLF.

\section{DISCUSSION}

Our findings suggest that the increase of $\mathrm{MD}$ in UF, ILF, and MLF of the right temporal lobe and the parathalamic white matter, the fornix and the posterior cingulate of the right mesial brain region is associated with increased apathy level. This finding is relevant, because an increase of MD indicates a loss of anisotropy related to abnormalities in the integrity of the tissue microstructure of the brain white matter [33, 34]. Furthermore, smaller areas of the left SLF I and 
Table 1

Sociodemographic, clinical, and neuropsychological characteristics of the 20 a-MCI patients

\begin{tabular}{|c|c|c|}
\hline Characteristics & $\begin{array}{l}\text { Raw values mean } \pm \mathrm{SD} \text {, or number and } \% \\
\text { (Correlations with DAIR scores)* }^{*}\end{array}$ & Adjusted values mean \pm SD \\
\hline Age (years) & $68.8 \pm 6.7$ & \\
\hline Educational level (years) & $8.8 \pm 3.9$ & \\
\hline Gender male: $\mathrm{n}(\%)$ & $6(30 \%)$ & \\
\hline Amnestic-MCI subtype & $\begin{aligned} 8 & =\text { single domain } \\
12 & =\text { multiple domain }\end{aligned}$ & \\
\hline DAIR score (Apathy diagnosis) & $\begin{array}{c}0.6 \pm 0.7 \\
(n=2 ; 10 \%)\end{array}$ & \\
\hline CERAD Dysphoria & $7.3 \pm 4.6$ & \\
\hline MMSE & $\begin{array}{c}27.2 \pm 2.3 \\
(r=-0.385 ; p=0.1994)\end{array}$ & $26.2 \pm 2.6$ \\
\hline RIR & $\begin{array}{c}28.6 \pm 7.9 \\
(r=-0.171 ; p=0.5854)\end{array}$ & $33.6 \pm 7.8$ \\
\hline RDR & $\begin{array}{c}4.1 \pm 3.4 \\
(r=-0.462 ; p=0.1144)\end{array}$ & $5.3 \pm 3.6$ \\
\hline RPM & $\begin{array}{c}22.4 \pm 4.6 \\
(r=0.315 ; p=0.3024)\end{array}$ & $24.8 \pm 4.5$ \\
\hline CROP & $\begin{array}{c}28.4 \pm 4.9 \\
(r=0.520 ; p=0.0685)\end{array}$ & $29.7 \pm 4.7$ \\
\hline DRROP & $\begin{array}{c}6.2 \pm 5.2 \\
(r=-0.329 ; p=0.2806)\end{array}$ & $9.5 \pm 5.3$ \\
\hline PVF & $\begin{array}{c}30.7 \pm 13.1 \\
(r=-0.272 ; p=0.3768)\end{array}$ & $34.3 \pm 12.1$ \\
\hline CFT & $\begin{array}{c}16.9 \pm 4.7 \\
(r=-0.088 ; p=0.7791)\end{array}$ & $\longrightarrow$ \\
\hline SCT & $\begin{array}{c}14.3 \pm 7.4 \\
(r=0.259 ; p=0.4028)\end{array}$ & $16.3 \pm 7.4$ \\
\hline $\mathrm{CD}$ & $\begin{array}{c}9.0 \pm 2.0 \\
(r=0.478 ; p=0.1000)\end{array}$ & $9.7 \pm 1.8$ \\
\hline CDL & $\begin{array}{c}63.4 \pm 4.8 \\
(r=0.298 ; p=0.3314)\end{array}$ & $64.3 \pm 4.5$ \\
\hline ST interference time & $\begin{array}{c}55.5 \pm 23.7 \\
(r=-0.143 ; p=0.6495)\end{array}$ & $\longrightarrow$ \\
\hline IVM & $\begin{array}{c}19.1 \pm 2.5 \\
(r=0.481 ; p=0.0972)\end{array}$ & $20.0 \pm 2.3$ \\
\hline MWCST achieved categories & $\begin{array}{c}5.6 \pm 0.6 \\
(r=0.197 ; p=0.5288)\end{array}$ & $\longrightarrow$ \\
\hline MWCST perseverative errors & $\begin{array}{c}2.3 \pm 2.4 \\
(r=-0.029 ; p=0.9262)\end{array}$ & $\longrightarrow$ \\
\hline MWCST non perseverative errors & $\begin{array}{c}2.4 \pm 2.4 \\
(r=-0.124 ; p=0.6931) \\
\end{array}$ & \\
\hline \multicolumn{3}{|c|}{$\begin{array}{l}\text { DAIR=Dementia Apathy Interview and Rating, MMSE=Mini Mental State Examination, RIR=Rey's } 15 \text {-word } \\
\text { Immediate Recall, RDR=Rey's } 15 \text {-word Delayed Recall, RPM=Raven's Progressive Matrices'47, CROP=Copy } \\
\text { of Rey-Osterrieth picture, DRROP=Delayed Recall of Rey-Osterrieth picture, PVF=Phonological Verbal Fluency, } \\
\text { CFT=Category Fluency Test, SCT =Sentence Construction Test, CD =Copy Drawings, CDL=Copy Drawings } \\
\text { with Landmarks, ST =Stroop Test, IVM=Immediate Visual Memory, MWCST=Modified Wisconsin Card Sorting } \\
\text { Test, SD=standard deviation. }\end{array}$} \\
\hline
\end{tabular}

III branches, the right SLF III branch, the left cingulum and the left ILF were also involved in this phenomenon. The associated decrease of FA in the same areas (except for the MLF) confirms that these areas were both microstructurally disrupted and disconnected.

All six larger white matter regions are likely involved in motivational processes and in emotional responses to stimuli. In fact, the UF, which occupies the white matter of the orbital and medial frontal structures and connects the temporal and prefrontal areas, is thought to play a crucial role in the regulation of emotional responses and in attaching emotional valence to visual information $[35,36]$. Furthermore, Fuji and colleagues [37] found that changes in the UF microstructure of a-MCI subjects were not only associated with memory impairment but also with emotional processing impairment, specifically emotional facial recognition. The ILF, which constitutes the association sys- 
tem of ventral visual pathways in the occipito-temporal cortices, is involved in emotional processing [35]; its disruption causes agnosia and prosopagnosia [36]. The MLF, which is situated in the white matter of the caudal inferior parietal lobule and links several high-level association and paralimbic cortical areas (e.g., the parietal lobule, the caudal cingulate gyrus, the parahippocampal gyrus, and the prefrontal cortex), is thought to play a role in linguistic processing of information dealing with spatial organization, memory, and motivational valence [36]. However, the MLF finding disappeared after depression severity correction and was not accompanied by FA reduction, indicating a nonspecific marginal role of this fasciculus in apathy pathogenesis in a-MCI.

As for the mesial brain region white matter, previous studies [38,39] described abnormalities located in the thalamus and the posterior cingulate in patients with MCI (it is to consider that posterior cingulate results, as MLF results, disappeared after depression severity correction). Our results also indicate an association between the fornix microstructure and apathy level in MCI patients. In fact, the fornix is an important component of memory and emotional circuitries, namely Papez's circuit, and its cholinergic fibers project to the hippocampus and anterior thalamic nuclei [40]. Thus, it is reasonable that increased white matter microstructural damage in the fornix causes impaired motivational and emotional processing and affects apathy level.

Although the main results were found in the right hemisphere, smaller white matter areas of the left hemisphere were also associated with apathy level. This indicates that apathy level is mainly associated with right hemisphere misconnection, which, to a lesser degree, extends also to the left hemisphere.

Limitations have to be acknowledged. Voxel-based analysis is prone to partial volume averaging and registration artefacts due to atrophy. In our study, however, partial volume effects were expected to play a minor role because the applied smoothing technique was proved to maintain tissue boundaries [19]. Although registration artefacts cannot be ruled out, they were limited by excluding those subjects whose excessive atrophies impeded correct registration and normalization. Another issue which may limit our results is the limited number of patients recruited in the study ( $n=$ 20 ), with only two patients considered as apathetic and a relatively low mean apathy score on the DAIR scale.

In conclusion, this is the first attempt to investigate the association between white matter microstructural damage and apathy level in a-MCI patients. In this study, we found that widely distributed alterations of white matter microstructure and connection are associated with apathy level. Indeed, these results contribute to clarifying the neural mechanisms underlying apathy. Future investigation of the correlates of apathy in other populations, such as depressed or demented subjects, and of the three main dimensions of apathy (lack of interest, lack of initiative and emotional blunting) is necessary. Finally, a categorical distinction between apathetic and non apathetic patients would be very useful in future studies on this topic.

\section{ACKNOWLEDGMENTS}

This research was supported by RC 05-06-07-0809/A and RF 06.71.8, PS 05.17, ISS.16 grants from the Italian Ministry of Health.

Authors' disclosures available online (http://www.jalz.com/disclosures/view.php?id=274).

\section{REFERENCES}

[1] Geda YE, Roberts RO, Knopman DS, Petersen RC, Christianson TJ, Pankratz VS, Smith GE, Boeve BF, Ivnik RJ, Tangalos EG, Rocca WA (2008) Prevalence of neuropsychiatric symptoms in mild cognitive impairment and normal cognitive aging: population-based study. Arch Gen Psychiatry 65, 1193-1198.

[2] Cummings JL (1997) The Neuropsychiatric Inventory: assessing psychopathology in dementia patients. Neurology 48 , S10-16.

[3] Spalletta G, Baldinetti F, Buccione I, Fadda L, Perri R, Scalmana S, Serra L, Caltagirone C (2004) Cognition and behaviour are independent and heterogeneous dimensions in Alzheimer's disease. J Neurol 251, 688-695.

[4] Palmer K, Di Iulio F, Varsi AE, Gianni W, Sancesario G, Caltagirone C, Spalletta G (2010) Neuropsychiatric predictors of progression from amnestic-mild cognitive impairment to Alzheimer's disease: the role of depression and apathy. $J$ Alzheimers Dis 20 175-183.

[5] Robert PH, Berr C, Volteau M, Bertogliati-Fileau C, Benoit M, Guerin O, Sarazin M, Legrain S, Dubois B (2008) Importance of lack of interest in patients with mild cognitive impairment. Am J Geriatr Psychiatry 16, 770-776.

[6] Teng E, Lu PH, Cummings JL (2007) Neuropsychiatric symptoms are associated with progression from mild cognitive impairment to Alzheimer's disease. Dement Geriatr Cogn Disord 24, 253-259.

[7] Vicini Chilovi B, Conti M, Zanetti M, Mazzu I, Rozzini L, Padovani A (2009) Differential impact of apathy and depression in the development of dementia in mild cognitive impairment patients. Dement Geriatr Cogn Disord 27, 390-398.

[8] Benoit M, Clairet S, Koulibaly PM, Darcourt J, Robert PH (2004) Brain perfusion correlates of the apathy inventory dimensions of Alzheimer's disease. Int J Geriatr Psychiatry 19, 864-869. 
[9] Craig AH, Cummings JL, Fairbanks L, Itti L, Miller BL, Li J, Mena I (1996) Cerebral blood flow correlates of apathy in Alzheimer disease. Arch Neurol 53, 1116-1120.

[10] Holthoff VA, Beuthien-Baumann B, Kalbe E, Ludecke S, Lenz O, Zundorf G, Spirling S, Schierz K, Winiecki P, Sorbi S, Herholz K (2005) Regional cerebral metabolism in early Alzheimer's disease with clinically significant apathy or depression. Biol Psychiatry 57, 412-421.

[11] Migneco O, Benoit M, Koulibaly PM, Dygai I, Bertogliati C, Desvignes P, Robert PH, Malandain G, Bussiere F, Darcourt J (2001) Perfusion brain SPECT and statistical parametric mapping analysis indicate that apathy is a cingulate syndrome: a study in Alzheimer's disease and nondemented patients. Neuroimage 13, 896-902.

[12] Robert PH, Darcourt G, Koulibaly MP, Clairet S, Benoit M, Garcia R, Dechaux O, Darcourt J (2006) Lack of initiative and interest in Alzheimer's disease: a single photon emission computed tomography study. Eur J Neurol 13, 729-735.

[13] Apostolova LG, Akopyan GG, Partiali N, Steiner CA, Dutton RA, Hayashi KM, Dinov ID, Toga AW, Cummings JL, Thompson PM (2007) Structural correlates of apathy in Alzheimer's disease. Dement Geriatr Cogn Disord 24, 91-97.

[14] Bruen PD, McGeown WJ, Shanks MF, Venneri A (2008) Neuroanatomical correlates of neuropsychiatric symptoms in Alzheimer's disease. Brain 131, 2455-2463.

[15] Petersen RC, Smith GE, Waring SC, Ivnik RJ, Tangalos EG, Kokmen E (1999) Mild cognitive impairment: clinical characterization and outcome. Arch Neurol 56, 303-308.

[16] Cox RW, Hyde JS (1997) Software tools for analysis and visualization of fMRI data. NMR Biomed 10, 171-178.

[17] SPM - Statistical Parametric Mapping, http://www.fil.ion. ucl.ac.uk/spm/, Last modified Date: 2009/04/02 Accessed on March 25, 2008

[18] Horsfield MA (1999) Mapping eddy current induced fields for the correction of diffusion-weighted echo planar images. Magn Reson Imaging 17, 1335-1345.

[19] Moraschi M, Hagberg G, Di Paola M, Spalletta G, Maraviglia B, Giove F (2010) The smoothing that does not blur: effects of the anisotropic approach for evaluating Diffusion Tensor Imaging data in the clinic. J Magn Reson Imaging 31, 690-697.

[20] Camara E, Bodammer N, Rodriguez-Fornells A, Tempelmann C (2007) Age-related water diffusion changes in human brain: a voxel-based approach. Neuroimage 34, 1588-1599.

[21] Talairach J, Tournoux P (1988) Co-Planar Stereotactic Atlas of the Human Brain, New York.

[22] Carlesimo GA, Caltagirone C, Gainotti G (1996) The Mental Deterioration Battery: normative data, diagnostic reliability and qualitative analyses of cognitive impairment. The Group for the Standardization of the Mental Deterioration Battery. Eur Neurol 36, 378-384.

[23] Osterrieth P (1944) Le test de copie d'une figure complexe: Contribuition à l'étude de la perception et de la mèmoire. Arch Psychol 30, 286-350.

[24] Rey A (1941) L'examen psychologique dans les cas d'encéphalopatie traumatique. Arch Psychol 28, 286-340.

[25] Lucas JA, Ivnik RJ, Smith GE, Bohac DL, Tangalos EG, Graff-
Radford NR, Petersen RC (1998) Mayo's older Americans normative studies: category fluency norms. J Clin Exp Neuropsychol 20, 194-200.

[26] Stroop J (1935) Studies of interferences in serial verbal reactions. J Exp Psychol 18, 643-662.

[27] Heaton R, Chelune G, Talley J, Kay C, Curtiss G (1993) Wisconsin card sorting test. Manual, Psychological Assessment Resources, Odessa, FL.

[28] Strauss ME, Sperry SD (2002) An informant-based assessment of apathy in Alzheimer disease. Neuropsychiatry Neuropsychol Behav Neurol 15, 176-183.

[29] Jacobs D, Staruss M, Patterson M, Mack J (1998) Characterization of depression in Alzheimer's disease by CERAD Behavior Rating Scale for Dementia (BRSD). Am J Geriatr Psychiatry Winter 6(1), 53-58.

[30] Rose SE, Janke AL, Chalk JB (2008) Gray and white matter changes in Alzheimer's disease: a diffusion tensor imaging study. J Magn Reson Imaging 27, 20-26.

[31] Benjamini Y, Hochberg Y (1995) Controlling the false discovery rate: a practical and powerful approach to multiple testing. J R Stat Soc Series B Stat Methodol 57, 289-300.

[32] Schmahmann JD, Pandya DN (2006) Fiber pathways of the brain, Oxford University Press, New York.

[33] Chua T, Wen W, Slavin M, Sachdev P (2008) Diffusion tensor imaging in mild cognitive impairment and Alzheimer's disease: a review. Curr Opin Neurol 21, 83-92.

[34] Le Bihan D, van Zijl P (2002) From the diffusion coefficient to the diffusion tensor. NMR Biomed 15, 431-434.

[35] Catani M, Jones DK, Donato R, Ffytche DH (2003) Occipitotemporal connections in the human brain. Brain 126, 20932107.

[36] Schmahmann JD, Smith EE, Eichler FS, Filley CM (2008) Cerebral white matter: neuroanatomy, clinical neurology, and neurobehavioral correlates. Ann N Y Acad Sci 1142, 266-309.

[37] Fujie S, Namiki C, Nishi H, Yamada M, Miyata J, Sakata D, Sawamoto N, Fukuyama H, Hayashi T, Murai T (2008) The role of the uncinate fasciculus in memory and emotional recognition in amnestic mild cognitive impairment. Dement Geriatr Cogn Disord 26, 432-439.

[38] Medina D, DeToledo-Morrell L, Urresta F, Gabrieli JD, Moseley M, Fleischman D, Bennett DA, Leurgans S, Turner DA, Stebbins GT (2006) White matter changes in mild cognitive impairment and AD: A diffusion tensor imaging study. Neurobiol Aging 27, 663-672.

[39] Rose SE, McMahon KL, Janke AL, O’Dowd B, de Zubicaray G, Strudwick MW, Chalk JB (2006) Diffusion indices on magnetic resonance imaging and neuropsychological performance in amnestic mild cognitive impairment. J Neurol Neurosurg Psychiatry 77, 1122-1128.

[40] Copenhaver BR, Rabin LA, Saykin AJ, Roth RM, Wishart HA, Flashman LA, Santulli RB, McHugh TL, Mamourian AC (2006) The fornix and mammillary bodies in older adults with Alzheimer's disease, mild cognitive impairment, and cognitive complaints: a volumetric MRI study. Psychiatry Res 147, 93103. 